Revista de Economia Política, vol. 33, no 3 (132), pp. 538-550, julio-septiembre/2013

\title{
La estructura económica del (sub)desarrollo y el equilibrio general o ¿qué ocurrió con la teoría del desarrollo y con las estructuras económicas?
}

FIDEL AROCHE REYES

Formerly the concept of economic development involved transforming the productive structures in order to employ the population in higher productivity activities, so that welfare improved. Development implied that economic systems followed development paths (not always in equilibrium) in order to reach more desirable welfare results: Equilibrium was not the main target. More recently, economic strategies emphasize reaching growth within equilibrium paths, thus, preserving economic structures. The latter vision yields incompatible results with the former. This paper revises some issues concerning structural change versus equilibrium targets as a means to reach development.

Keywords: Development; economic structure; equilibrium, disequilibrium.

JEL Classification: O10; O14; O40.

\section{INTRODUCCIÓN}

El desarrollo económico es un tema ausente en la discusión académica y económica general, a partir de que en 1981 el súbito incremento de las tasas de interés en los mercados internacionales de fondos se tradujo en inflación, desequilibrio fiscal, desempleo y contracción del producto bruto para muchos países no desarrollados. El problema del desarrollo dejó de ser prioritario, para dar espacio a los problemas urgentes de desequilibrio macroeconómico, el cumplimiento con los

\footnotetext{
* Profesor, Facultad de Economía e Instituto de Investigaciones Económicas, Universidad Nacional Autónoma de México. E-mail: aroche@servidor.unam.mx. Agradezco a Oscar Ugarteche y a un dictaminador anónimo por sus observaciones a versiones anteriores de este trabajo. Los errores supervivientes son mi entera responsabilidad. Submitted: 15/August/2011; Approved: 07/August/2012.
} 
compromisos relativos a la deuda pública y rescatar empresas públicas y privadas. Hasta entonces el sentido común coincidía en que los países no desarrollados deberían crecer rápidamente e industrializarse, modificando sus estructuras productivas a fin de resolver problemas económicos y sociales; cuando crece la industria manufacturera, crea ipso facto empleos con mayor productividad y mejores remuneraciones y los trabajadores tienen capacidad de aprender y se crea también un mercado interno dinámico; es decir, se crean círculos virtuosos que aceleran el crecimiento. Después del año 2000 solo algunos países del Asia han continuado por esta vía, sustituyendo exportaciones manufactureras en general por exportaciones de alta tecnología, mientras los conceptos de desarrollo y subdesarrollo han dado paso a categorías como "países emergentes" y al estudio de la pobreza.

En el plano de la teoría económica, a partir de la década de 1970, ganó terreno la idea de que no es preciso que las autoridades regulen la actividad económica, ni que las autoridades intervengan activamente para resolver desequilibrios o para practicar políticas de fomento a actividades económicas específicas. El sistema económico solo requiere de un marco legal y uno de política macroeconómica estable, en donde producir resultados de equilibrio que ponen en concierto las acciones de los agentes individuales. Estos resultados serán óptimos y por lo tanto proporcionarán el máximo nivel de bienestar posible para cada individuo, dados los recursos y su distribución.

De acuerdo con este punto de vista el subdesarrollo es una condición en la que la economía produce resultados de equilibrio subóptimo (Bauer, 1972; Hoff, 2001); en particular, los ingresos son inferiores a aquellos que resultarían del equilibrio eficiente, de allí la pobreza de una alta proporción de la población. Ello ocurre principalmente porque en los países pobres las autoridades practican políticas inadecuadas y existen instituciones viciadas; ambas se refuerzan unas a otras y responden a estructuras de poder autoritarias de las que la burocracia se beneficia a costa del bienestar social. En estos países la burocracia y los hacedores de política económica defienden sus propios intereses y perpetúan prácticas y costumbres que impiden que la competencia entre los agentes lleve a la economía al equilibrio óptimo (Krugman, 1974).

Entonces la política económica debe reducirse a eliminar los obstáculos que impiden el funcionamiento adecuado de las instituciones económicas, de modo que los agentes puedan tomar las decisiones eficientes y el sistema incremente los ingresos de la población. En todo caso, las políticas sociales se encargarán de dotar a los pobres con los recursos que les capaciten para participar en la economía. Las reformas económicas generalizadas harán que desaparezcan las instituciones donde la burocracia se refugia y hará espacio para que los recursos se asignen de manera eficiente en el sentido de Pareto (Mas Collel, et al., 1995) y las empresas crezcan. No existe entonces un problema de subdesarrollo económico, sino la prevalencia de bajos ingresos y ausencia de crecimiento.

No obstante, en otro orden de ideas el subdesarrollo ha sido caracterizado por ser un síndrome que va más allá de la pobreza; no es en ese sentido un problema que se soluciona con el incremento de los ingresos, porque éstos no crecerán sufi- 
cientemente ni se distribuirán adecuadamente. Por ejemplo, el subdesarrollo incluye la marginación y la exclusión de segmentos de la población, de regiones o de actividades económicas; asimismo se ha señalado la existencia de disparidades profundas entre las ramas o los sectores económicos (Sunkel, 1972). Entonces, la solución del síndrome del subdesarrollo requiere cambiar la senda de expansión de la economía y redistribuir los recursos y el producto entre los agentes, las ramas y las regiones. Se trata en principio de una solución de desequilibrio. La expansión equilibrada del producto en el mejor de los casos producirá mayores ingresos, pero no atenderá los problemas estructurales del subdesarrollo (Prebisch, 1949). Luego, el crecimiento económico equilibrado no es equiparable con el desarrollo.

Como se ha anotado arriba, la idea del subdesarrollo ha caído en desuso en el análisis económico, junto con la idea de que deban atacarse los problemas de las economías, en el sentido de emprender una senda de desarrollo. No obstante, muchos de los fenómenos económicos prevalecientes en algunos países menos desarrollados, después de tres décadas de reformas generalizadas en búsqueda del crecimiento sostenido y en equilibrio eficiente invitan a reconsiderar la relevancia empírica de este concepto. Este documento justamente repasa algunas ideas acerca del desarrollo que se discutieron durante los años 1950, a partir de una preocupación sobre la estructura productiva y las consecuencias que su evolución puede tener sobre el bienestar de la población.

\section{LA TEORÍA DEL SUBDESARROLLO}

El modelo de equilibrio general con crecimiento equilibrado de Gustav Cassel (1918) y la solución propuesta a este modelo por John (Janos) von Neumann (1937) han sido muy influyentes en la discusión teórica sobre la existencia del equilibrio y sobre el crecimiento. En ambos modelos el sistema económico alcanza el equilibrio con crecimiento uniforme, si bien en el primero se trata del resultado de una deducción lógica y en el otro las condiciones para la demostración de la existencia del equilibrio son bastante restrictivas, si bien vale recordar que el propósito del modelo de von Neuman no es su aplicación empírica. Así, todas las ramas de la economía se expanden a una misma tasa uniforme, de modo que el sistema de precios en ambos modelos, una vez determinado, es estable y si son crecientes los recursos, se distribuyen también de manera uniforme entre los sectores. Ningún sector crece fuera de esta tasa de equilibrio, no existe desempleo de los recursos y su productividad es siempre de equilibrio e igual a sus remuneraciones. El resultado es siempre eficiente.

A partir de la década de 1930, a la luz de la depresión económica mundial, de los problemas económicos de la postguerra en la década siguiente, además de los procesos de descolonización de amplias zonas del mundo en los años 1950 y 1960, se hizo evidente que las diversas economías tenían características distintas; algunas eran predominantemente productoras y exportadoras de bienes primarios y otras producían bienes manufacturados; en algunas el desempleo era resultado de las 
fluctuaciones del nivel de actividad económica, en otras el desempleo era una condición permanente: tenían "exceso de mano de obra", que se disfrazaba en el campo, donde la mano de obra mostraba baja productividad. El desempleo urbano se disfrazaba también bajo la forma de empleo precario o bajo estilos señoriales, para emplear el lenguaje de Lewis (1954). En estos países, las remuneraciones estarían más asociadas con las costumbres que con la productividad, como ocurre en una economía capitalista plena.

Algunos países subdesarrollados también presentaban concentración de la propiedad y de los ingresos, mientras que las estructuras sociales impedían la movilidad social y a veces, incluso la movilidad física de los trabajadores, quienes estaban ligados a la tierra. Las estructuras políticas respondían a estas circunstancias y las políticas económicas no podían modificar la realidad. De este modo el crecimiento no sería suficiente para garantizar a toda la población el acceso a mejores niveles de bienestar. En el plano económico, si bien la producción se dirigía frecuentemente al mercado internacional, la lógica al interior de las explotaciones no siempre obedecía a los criterios capitalistas de optimización.

La teoría del desarrollo de los años 1940 y 1950 percibe entonces que el subdesarrollo es un fenómeno que incide en todas las esferas al interior de las sociedades no desarrolladas. Prebisch (1949) agregaría que la manera en que las economías se insertan en los mercados internacionales también responde a la condición de desarrollo o de subdesarrollo. En el primer caso las economías compiten a partir de la productividad creciente y los costes de producción decrecientes, merced del carácter de la misma competencia capitalista, mientras que las exportaciones incluyen bienes manufacturados y las economías se benefician de la introducción de innovaciones. En el segundo caso, los países exportan a partir de sus dotaciones de recursos naturales y por lo tanto, no son capaces de apropiarse de los beneficios de la competencia o de la introducción de innovaciones. De este modo, los países no desarrollados son incapaces de incidir en sus condiciones de inserción en los mercados internacionales. La caída de los términos de intercambio, no obstante su celebridad es menos importante para determinar la condición de subdesarrollo que las condiciones en que se producen las exportaciones, como diría Furtado (Mallorquín, 1999). La solución a los problemas del subdesarrollo implica entonces la transformación de las sociedades a partir de la modernización de sus sistemas productivos (Sunkel, 1972). Ello demanda la intervención del estado en la mayor parte de los casos, porque en estas sociedades no otras existen instituciones con la energía suficiente para impulsar la modernización de las economías y su reinserción en los mercados internacionales. Es decir, la intervención pública en la economía es un resultado de la necesidad de desarrollo; los mercados no existen o no están constituidos plenamente y no es posible dejar a ellos la solución del subdesarrollo.

Los casos más conocidos en América son por supuesto el de la Argentina, el Brasil y México, donde se instituyeron regímenes que emprendieron estrategias de desarrollo con el propósito explícito de transformar las estructuras económicas y sociales. La industrialización transforma la estructura productiva y proporciona empleo de mayor productividad; en México, la reforma agraria también tiene el 
objetivo de modernizar al sector rural, al destruir la base de poder de la oligarquía terrateniente, mientras los pequeños agricultores pueden convertirse en consumidores de bienes industriales. En la Argentina y el Brasil el crecimiento industrial haría desaparecer la presión sobre la propiedad rural, a la manera de los países europeos, donde no se producen reformas agrarias como condición de la modernización general de la sociedad. No obstante, existen otros casos donde el estado también asumió papeles activos para promover la industrialización, como medio de alcanzar el desarrollo económico a partir de la segunda postguerra, como la India, Corea del Sur, Turquía, Israel e incluso, algunos países europeos (Chang, 2002). Por supuesto, el éxito relativo de estas diversas industrializaciones no puede atribuirse solamente a la intervención del estado, puesto que existen también casos de industrialización comandada por el estado que no produjeron desarrollo, como en el África (Petiteville, 2002).

Paul Rosenstein-Rodan (1943) reflexiona sobre las condiciones económicas de Europa Oriental y plantea que el desempleo abierto o encubierto de las sociedades subdesarrolladas obedece a que la estructura económica es incapaz de ofrecer empleo a la población - si bien no emplea esta terminología - al mismo tiempo, esta población no tiene las habilidades para emplearse. Así, es necesario crear nuevas actividades productivas donde la mano de obra subempleada o desempleada pueda encontrar empleo remunerado de acuerdo con una mayor productividad. La alternativa es llevar a la población redundante a países donde existen oportunidades de empleo, es decir, la emigración masiva hacia los países prósperos que ofrecen empleo, como ocurre en la actualidad. Por el contrario, el modelo neoclásico de comercio internacional predice que la propia abundancia de mano de obra en estos países los conducirá a mayores niveles de empleo con mayores niveles de remuneración, si aquellos exportan los bienes para los que tienen mejores condiciones de producción. El modelo Heckscher-Ohlin no prevé la existencia de barreras institucionales que impiden el funcionamiento de los mercados ni que impiden la convergencia de los salarios en la economía mundial.

En el lenguaje de la teoría neoclásica, la propuesta de Rosenstein-Rodan requiere de la participación de un "planificador central" decidido a que la economía produzca resultados de equilibrio más deseables al inicial, de acuerdo con sus preferencias, que representan las de la sociedad en su conjunto. Es decir, el planificador debe redistribuir los recursos iniciales de la sociedad a fin de que sea posible la creación simultánea de un conjunto de nuevas actividades productivas. La nueva producción se dirige al mercado interno, en principio porque la población trabajadora en la manufactura requiere de satisfactores en cantidades y calidades distintas a la situación anterior de subempleo rural, también porque la nueva producción requiere de mercados y en las primeras fases no será necesariamente competitiva en el mercado internacional. Asimismo, la población que permanece en el campo ve elevarse su productividad e ingreso cuando la población excedente emigra al sector manufacturero, de modo que también se transforma en consumidora de bienes industriales. Entonces, la dinámica del sistema se beneficia de que los nuevos trabajadores industriales gocen de mayores salarios, acordes con su productividad 
acrecentada por trasladarse hacia las nuevas manufactureras. Esta propuesta se redondea con la idea de que las nuevas actividades productivas sean complementarias unas de otras, a fin de que cada una sea beneficiaria de las externalidades que surgen de un esfuerzo coordinado entre diversas actividades y empresas. Si bien Rosenstein-Rodan recoge la noción de crecimiento equilibrado, claramente propone el cambio de la estructura económica en los países con exceso de mano de obra, lo que es distinto a una solución para trasladar al sistema económico de un equilibrio con menor nivel de actividad hacia otro equilibrio, sin crear nuevas actividades económicas, como sugieren los autores más modernos asociados con la economía neoclásica. Por ejemplo, Hoff (2001) no acepta la existencia de una estructura económica incapaz de llegar a equilibrios óptimos (sin desempleo). La propuesta de Rosenstein-Rodan es de crear una situación de desequilibrio inicial para transformar a la estructura productiva, a fin de llegar a un punto de equilibrio más deseable.

Reconociendo que la inversión se dirige espontáneamente de manera diferenciada entre los sectores - ya que responde a la rentabilidad real y a la esperada - y que mediante la inversión se incorpora el progreso técnico a las actividades productivas, la teoría del crecimiento desequilibrado reconoce que los sectores económicos crecerán a tasas diferenciadas, por lo que las estructuras económicas se modifican autónomamente a lo largo del tiempo. Löwe (1926 y 1976) propone que el sistema económico no necesariamente llega al equilibrio, sino que, por el contrario, los distintos sectores siguen dinámicas diferenciadas, lo cual, junto con la incorporación del progreso técnico, genera que las economías sigan patrones cíclicos de comportamiento. A partir de estos postulados Hirschman (1958) postula que el desarrollo es un proceso desequilibrado y justamente ese carácter es un elemento que si los encargados de la política económica saben emplear, llevará a la economía a ritmos mayores de crecimiento, si las ramas con mayor capacidad de incorporación del progreso técnico y de propagación de los impulsos al crecimiento se expanden a mayor velocidad. La política económica entonces debe también promover los desequilibrios para generar brechas a favor de los sectores más dinámicos.

El desarrollo se asocia entonces con el desequilibrio sectorial que supone la construcción permanente de una estructura económica diversificada, compleja e integrada vertical y horizontalmente, donde existen múltiples actividades económicas que se relacionan entre sí de múltiples formas, determinadas en última instancia por la tecnología que emplean las actividades en la producción. Esta estructura debe incluir sectores de diversos niveles tecnológicos, así como sectores innovadores y de crecimiento dinámico. La diversidad estructural debe ser compatible con un perfil de especialización definido, si es que la economía no es autárquica (Balassa, 1969).

En efecto los determinantes del desarrollo económico son también fuerzas que desequilibran al sistema. Entre estos determinantes aparecen por ejemplo el crecimiento desequilibrado de las ramas y sectores, el desarrollo de nuevas actividades productivas, la inversión productiva, la innovación y otros. El modelo de crecimiento equilibrado de Cassel y su moderna solución en el modelo de Arrow y Debreu 
no son capaces de incorporar estos fenómenos en el análisis. Las matemáticas de la dinámica económica con $l$ bienes y $n+m$ agentes $(n$ productores y $m$ consumidores) son ya muy complejas y uno de los supuestos de estos modelos es que los agentes tienen conocimientos relevantes sobre los bienes, las tecnologías y los precios. Un sector que crece más rápidamente supone que es más rentable: no habría razones para que ningún productor permaneciera en la producción de sectores rezagados o mejor, no deberían haber sectores rezagados, puesto que el proceso de competencia debería corregir todas las disparidades.

\section{LA ESTRUCTURA DEL DESARROLLO}

La estructura económica que caracterizaba a los países subdesarrollados en los años 1940 era dominada por las actividades rurales o mineras, que producían bienes para la exportación, donde las empresas podían mantener o no relaciones capitalistas - o modernas - con sus trabajadores y con su entorno económico. Los bienes manufacturados eran mayormente importados, si bien en algunos países existían segmentos de la economía donde se encontraban empresas manufactureras modernas, produciendo para el mercado interno principalmente; el tamaño de estos segmentos y el grado de modernidad de las relaciones económicas en las explotaciones para la exportación variaba en función del grado de modernización de las propias economías. De todos modos, como lo apuntan Rosenstein-Rodan (1943) y Lewis (1954), esta estructura no era capaz de emplear a la población, como tampoco lo habría sido creciendo sin modificaciones en sus proporciones fundamentales. Era necesario crear actividades manufactureras modernas, como las únicas capaces de crecer rápida y suficientemente, garantizando que los recursos fueran absorbidos plenamente por el sistema económico, alcanzando tasas de productividad competitivas, proporcionando niveles suficientes de ingresos, incluso a poblaciones grandes. La misma población trabajadora puede proporcionar la demanda necesaria para garantizar el crecimiento del sector.

En los países donde ocurrió la industrialización fue necesario trasladar recursos hacia las nuevas actividades productivas, donde tanto de los trabajadores como de los propios empresarios participaron en procesos de aprendizaje para adaptarse a las nuevas actividades. Ello no ocurrió sin costes de todo tipo y en particular propició movimientos migratorios al interior de los países, cuando la población se mudó a las actividades manufactureras. Asimismo, sin importar la ubicación geográfica o los resultados finales, en todos los países la industrialización ha supuesto que los estados hayan practicado políticas de industrialización específicas, lo cual ha implicado mayores niveles de intervención de lo que están dispuestos a reconocer algunos organismos económicos internacionales (Chang, 2003).

En los países que se industrializaron la producción se diversificó y se construyeron ramas productoras de bienes finales e intermedios que pudieron integrarse vertical y horizontalmente; es decir, el desarrollo mismo de la manufactura y la especialización de las ramas dio lugar a patrones de división del trabajo y de coo- 
peración entre ellas y al intercambio acrecentado de bienes. De este modo, el desarrollo económico se ha asociado con el mayor grado de complejidad de las estructuras productivas: la mayor especialización de las ramas y el mayor intercambio entre ellas da lugar a mayores niveles de desarrollo (Leontief, 1963). Los procesos productivos demandan insumos específicos en proporciones específicas, determinados por las tecnologías que cada rama emplea; en una economía más desarrollada las ramas son más especializadas y dependen en mayor medida de los insumos que adquieren de otros productores, lo cual a su vez ofrece mayores oportunidades para la creación de otras empresas especializadas, que a su vez se benefician de los intercambios que ocurren en la estructura productiva, la cual adquiere mayor nivel de complejidad. Este planteamiento supone que en los países grandes los insumos son de procedencia nacional, pero desde luego no es un planteamiento de autarquía ni de protección de actividades ineficientes, pero sigue la idea de las actividades "infantes", incapaces de competir sin los mecanismos de protección y donde se deben fomentar los procesos de aprendizaje para subsanar esta carencia.

Si los productores al interior de una región o de un país cooperan, generan economías externas a cada empresa y actividad, pero internas a la región o al país. Los productores pueden cooperar con sus proveedores de manera consiente y explícita o no, pero será más fácil de este modo que al interior de un país la producción se diversifique y los productores se integren de manera vertical y horizontal que cuando la totalidad de los insumos es importada. Por ejemplo, un productor puede innovar y hacer que su proveedor innove al mismo tiempo, al hacer un pedido de insumos: entre ambos crean nueva tecnología y los beneficios de esta cooperación se quedarán al interior del país. La importación de insumos solo asegura que en el corto plazo los costes de los productores finales serán menores a la situación cuando el productor nacional no ha aprendido a producir el insumo correcto y queda en duda dónde quedarán los beneficios de la innovación o de la creación de tecnología. De este modo, la integración de los productores y su cooperación propiciará que se creen mayores oportunidades de empleo para trabajadores altamente calificados y para el conocimiento que la población pueda adquirir en el sistema educativo.

La construcción de una estructura productiva compleja y diversificada avanzó de manera diferenciada entre los países que emprendieron procesos de industrialización a partir de la postguerra, con frecuencia siguiendo patrones de sustitución de importaciones con protección arancelaria y políticas de fomento industrial (Chang, 2003). En Europa y en Asia algunas de estas estructuras dieron lugar a perfiles más nítidos de especialización junto con grados de apertura mayores de las economías; la industrialización de las economías resolvió muchos de los problemas que caracterizan al subdesarrollo y modificó en muchos casos la manera en que estos países se insertan en la economía mundial, cuando pasaron a ser exportadores de manufacturas con tecnologías autóctonas y productores integrados.

En algunos otros países como los americanos la modernización de las estructuras económicas, sociales y políticas no se completó, de modo que luego de décadas de industrialización, los resultados de la industrialización en términos de la 
distribución de la propiedad y del ingreso no fueron los más deseables, mientras el subempleo no necesariamente desapareció ni se modernizaron completamente las actividades primarias. Es bien conocido el caso de que América es el continente donde el ingreso se haya particularmente concentrado. A modo de ejemplo, de acuerdo con datos de la Comisión Económica para América Latina (CEPAL, 2012), el coeficiente de Gini alcanza en la Argentina el 38\% en 2011, el 58\% en el Brasil y Colombia, 52 en Chile, 47 en el Perú, 42 en el Uruguay en 2009 y $52 \%$ en México en 2008. A modo de contraste, en dos de los países más igualitarios del mundo como Dinamarca y Suecia el coeficiente es de $25 \%$ en el 2000 (datos del Banco Mundial).

Al mismo tiempo, no siempre la industria mostró haber adquirido capacidades para competir en los mercados internacionales, de modo que las economías recientemente industrializadas continuaron con la exportación de bienes primarios, que financiaba las necesidades de importación de las manufacturas industriales. A este respecto Bruton (1998) señala que, por una parte, las políticas económicas no fomentaban las exportaciones manufactureras ni la competitividad de las economías, como quizá tampoco fomentaron la integración interna de los productores ni la creación de tecnologías o de marcas propias a los países, que son por cierto los segmentos productivos más rentables y de mayor productividad. De este modo los sectores manufactureros en muchos países mostraban déficit endémicos en cuenta corriente; estas industrializaciones pueden ser caracterizadas como incompletas. Sin embargo, Ocampo (2005) señala que hacia la década de 1980 las exportaciones de muchos países latinoamericanos comprendían una importante proporción de manufacturas con componentes nacionales (a diferencia de la industria de ensamblados con componentes importados) que a veces eran productos de empresas nacionales, no solo de transnacionales afincadas en los países exportadores. En efecto, las exportaciones manufactureras disminuyeron en varios países latinoamericanos como porcentaje del total, en el Brasil la proporción pasó de $51.9 \%$ en 1990 a 36.4 en 2010, en Colombia del $25.1 \%$ al 22.1, en Chile la proporción se mantuvo estable, del $10.9 \%$ al 10.4, en el Perú la disminución fue del $18.4 \%$ al 10.9 y en el Uruguay del $38.5 \%$ al 25.7. En la Argentina las exportaciones manufactureras ganaron importancia en ese mismo período, pasando del $29.1 \%$ al 32.2; en México las exportaciones manufactureras también han tenido una notable expansión, pasando del $43.3 \%$ del total en 1990 al $74.7 \%$ en 2010, sin embargo, según cifras oficiales del Instituto Nacional de Estadistica (INEGI), en 1990 el 40\% de tales exportaciones provenían de la industria que ensambla componentes importados y esa proporción llegó al 48\% en promedio entre 2000 y 2006.

Entre el fin de la década de 1970 los primeros años 1980 los países latinoamericanos cayeron en crisis simultáneamente, cuando las tasas de interés por sus deudas externas se elevaron súbita y significativamente, de modo que era imposible satisfacer los montos y los plazos de pago (Ugarteche, 1986). No obstante, la explicación de la crisis que ganó preminencia en muchos ámbitos sociales y políticos la atribuía a la corrupción en las esferas gubernamentales, la incapacidad administrativa del Estado y la mala asignación de recursos ocasionada por las políticas de 
fomento a la industrialización de espaldas a la disponibilidad inicial de los recursos (Krueger, 1974). De este modo, según tal punto de vista, la manufactura se orientaba de casi exclusiva hacia los mercados internos protegidos de tamaño limitado y donde la competencia no era suficiente para inducir a los empresarios a adoptar conductas eficientes, mientras que - sigue el argumento- el crecimiento exportador hubiera seguido una senda de crecimiento ilimitada, puesto que el mercado internacional es mucho mayor y más dinámico, mientras que observa una estructura competitiva, aún si los mercados nacionales son monopolistas u oligopolistas. La solución a la crisis incluía la privatización de las empresas públicas y la limitación a la intervención del Estado en asuntos económicos, como parte de las reformas mencionadas al principio del documento, además de procesos de ajuste severos, buscando alcanzar los equilibrios macroeconómicos y particularmente, reorientar a las economías hacia los mercados internacionales; el argumento era corregir las fallas de estas industrializaciones incompletas, reasignando los recursos y resolviendo también los problemas de balance de pagos (Sunkel y Griffiths-Jones, 1987).

Las reformas aplicadas con celo se tradujeron en la desestructuración de los mercados internos - en cambio de la integración de las economías a los mercados internacionales - y en el desmantelamiento de los aparatos industriales o de algunos de sus segmentos, suponiendo la imposibilidad de que las actividades manufactureras elevaran su productividad y se tornasen competitivas. En efecto, muchos países latinoamericanos muestran síntomas de desindustrialización similares a algunos países avanzados, aún antes de haber completado la industrialización. Por ejemplo siguiendo la base de datos de la CEPAL (2012), en la Argentina el peso de la industria (manufacturas más electricidad) en el PIB en 1990 era de $26.4 \%$ y en 2011 de $24.6 \%$, mientras los servicios elevaron su participación en 2 puntos porcentuales; en el Brasil la industria descendió de $23.2 \%$ al 20.4; en Colombia el descenso fue del $23.5 \%$ al 17.9 ; en Chile del $19.2 \%$ al 15.6; en el Uruguay el descenso fue del $24 \%$ al 17.3. En México el peso de la industria se mantuvo estable y en todos los casos los servicios incrementaron su peso en el Producto. En el Perú tanto la industria como los servicios retrocedieron como componentes del PIB, mientras la minería y la construcción incrementaron su presencia.

Simultáneamente las economías han disminuido su capacidad de crear suficientes fuentes de empleo, sobre todo para el trabajo calificado; como consecuencia, la población se ha refugiado en actividades informales y la participación de los salarios en el producto se ha reducido. Por ejemplo, de acuerdo con la CEPAL (2012) en la Argentina el 38\% de los ocupados urbanos se ubicaban en actividades de baja productividad (sector informal), ese porcentaje alcanzó el $41 \%$ en Brasil, $30 \%$ en Chile, $60 \%$ en Colombia, $45 \%$ en México, $58 \%$ en el Perú y $39 \%$ en el Uruguay.

Es decir, muchas de las empresas manufactureras expuestas súbitamente a la competencia no sobrevivieron o se transformaron de productoras a importadoras y comercializadoras de los bienes previamente producidos. La industria creada durante las décadas previas fue responsabilizada de todos los problemas económicos que presentaban los países y no se consideró posible su reconversión (Hirsch- 
man, 1992). Probablemente los consumidores serían los beneficiarios de precios reducidos y mayor variedad de la oferta, pero algunos de estos mismos consumidores perderían sus empleos. Las ganancias de la asignación más eficiente de los recursos quedan en entredicho cuando la población no tiene posibilidades de empleo de calidad, a menos que emigre, fenómeno que por supuesto ocurrió.

La quiebra masiva de empresas significó también la pérdida de recursos, conocimientos y capacidades productivas, puesto que los recursos invertidos no se recuperaron. Asimismo, las estructuras económicas han perdido complejidad a medida que las ramas se tornan más dependientes de las importaciones de insumos, los productores en muchos casos se limitan a ensamblar componentes importados, generando reducido valor agregado. En estos procesos no existe la posibilidad de integración de la economía, de que los agentes aprendan ni de que la productividad crezca. En algunos casos las economías han vuelto a depender básicamente de las exportaciones de bienes primarios y en otros, las exportaciones manufactureras de bienes ensamblados no tienen mayor relación con la economía interna, por lo que no aportan impulsos al crecimiento sostenido. Por el contrario, hay países donde las reformas han sido puestas en práctica con menor acuciosidad, lo cual ha permitido la supervivencia de segmentos manufactureros; existen también países donde la política económica no abandonó la promoción industrial totalmente, de modo que se ha reacomodado la estructura productiva, dando lugar a que las economías reorienten su perfil de especialización. Aquí más que probablemente el esfuerzo industrializador inicial ha tenido la posibilidad de sobrevivir y aun quizás sea posible la construcción de una estructura productiva para el desarrollo, diversificada e integrada.

\section{CONCLUSIONES}

A partir de los años 1950 algunos de los países han logrado superar la condición de subdesarrollo, como algunos países europeos, Corea del Sur e Israel, por nombrar algunos donde las estrategias de desarrollo consiguieron modernizar las estructuras económicas y sociales, elevando los niveles de ingreso y ofreciendo empleo de calidad a la población. No obstante, muchos otros países antes llamados subdesarrollados - y ahora emergentes - no han resuelto muchos de los problemas económicos básicos, de modo que se repiten fenómenos como el exceso de población (o el desempleo estructural) y los bajos ingresos, asociados con la prevalencia de actividades de baja productividad, mientras, la distribución del ingreso es inequitativa y - como fenómeno nuevo - muchos de estos países expulsan población, que emigra en busca de empleo y oportunidades. No obstante, la teoría del desarrollo reciente desconoce que estos fenómenos (y otros) derivan del hecho de que la propia estructura productiva es incapaz de ofrecer a la población empleo suficiente con alta productividad, que haga posible elevar los niveles de ingreso. En cambio, se buscan las causas de la pobreza y el subempleo en las características de la población aquejada. 
Por otro lado, la discusión se ha centrado en la presencia o ausencia del estado en los procesos de desarrollo como explicación de los resultados de los procesos de desarrollo más recientes. Asimismo, se atribuye al sector externo una capacidad especial para hacer desarrollar a las economías, ampliando las posibilidades de crecimiento de las empresas privadas de los países llamados "emergentes". Desde luego, las políticas económicas y la inversión del estado han sido determinantes en algunos casos de desarrollo ocurrido en las últimas décadas, pero sin duda, estos esfuerzos son más exitosos allí donde los las sociedades se han modernizado y han conseguido construir estructuras económicas complejas, gracias al desarrollo de nuevas actividades con mayor productividad, donde se ofrecen oportunidades de negocios y de empleo adecuadas para el tamaño de su población. Por otra parte, la mayor competitividad de los países ricos quizá sea un resultado del propio proceso de desarrollo y no necesariamente su causa fundamental.

En efecto, la competitividad de las empresas y de las economías puede explicarse por diversas causas, como la capacidad empresarial, el diseño de los productos, los servicios asociados con su venta y distribución. En una economía desarrollada la población educada y las empresas tienen un medio ambiente que facilita los procesos de aprendizaje y de competitividad.

La discusión del desarrollo entonces debe volver a la idea de que la estructura productiva prevaleciente en una economía le confiere unas capacidades particulares para atender las necesidades de su población. De este modo, las economías subdesarrolladas se diferencian de las desarrolladas en primer término por sus estructuras, de modo que en aquellas la ausencia de algunos mercados o el carácter que adquieren éstos impiden que se alcancen asignaciones de recursos deseables, más allá de que el nivel de ingreso es insuficiente para que la población alcance mayores niveles de bienestar. Solucionar los problemas del subdesarrollo requiere la construcción de una estructura productiva distinta.

\section{REFERENCIAS BIBLIOGRÁFICAS}

Balassa Bela (1969) "Development in an Open Economy: The Case of Norway", Oxford Economic Papers New Series, Vol. 21, No. 3, noviembre, pp. 344-359.

Bauer Peter (1972) Dissent on Development: Studies and Debates in Development Economics. Cambridge, Mass: Harvard University Press.

Bruton Henry (1998) “A Reconsideration of Import Substitution,” Journal of Economic Literature, Vol. XXXVI, No. 2, junio.

Cassel Gustav (1918) Theoretische Sozialökonomie. Traducido al castellano como Economía Social Teórica $2^{\circ}$ Edición, Madrid, M. Aguilar Editor, 1941.

CEPAL (2012) CEPALSTAT Bases de datos y publicaciones estadísticas http://websie.eclac.cl/infest/ ajax/cepalstat.asp?carpeta=estadisticas (consultado el 5 de junio de 2012)

Chacholiades, Miltiades (1993) Economía internacional, Santa Fe de Bogotá: Mc. Graw-Hill.

Chang Ha-Joon (2002) Kicking Away the Ladder - Development Strategy in Historical Perspective, Londres: Anthem Press.

Chang Ha-Joon (2003) "The East Asian Development Experience” en Ha-Joon Chang (Editor) Rethinking Development Economics” Londres: Anthem Press. 
Griffith-Jones, Stephany y Osvaldo Sunkel (1987) La crisis de la deuda y del desarrollo en América Latina: El fin de una ilusión, Buenos Aires: Grupo Editor Latinoamericano.

Hirschman Albert O. (1958) The Strategy of Economic Development. New Haven, Conec: Harvard, University Press.

Hirschman Albert O. (1992) "Industrialization and its manifold discontents: West, East and South" World Development, Vol. 20, No. 9, pp. 1225-1232.

Hoff Karla (2001) "Beyond Rosenstein-Rodan: The Modern Theory of Underdevelopment Traps" en Boris Pleskovic y Nicholas Ster (Editores) Proceedings of the Annual World Bank Conference on Development Economics, pp. 177-182. Julio 2001. http://www-wds.worldbank.org/external/default/WDSContentServer/WDSP/IB/2001/12/11/000094946_01112104010389/Rendered/PDF/ multi0page.pdf (15 de marzo, 2011).

Krugman Ann (1974) “The Political Economy of the Rent Seeking Society," American Economic Review, Vol. 64, No. 3, pp. 291-303.

Leontief Wassily (1963) “The Structure of Development” en Scientific American, vol. 209, pp. 148-166.

Lewis Arthur (1954) "Economic Development with Unlimited Supplies of Labour" en Manchester School of Economic and Social Studies, Vol. 22, pp. 139-1991.

Löwe Adolph (1926) “Wie ist Konjunturtheorie überhaupt möglich?” en Weltwirtschaftliches Archiv No. 24, pp. 165-97.

---- (1976) The Path of Economic Growth. Cambridge: Cambridge Universtity Press.

Mallorquín Carlos (1999) "La historia del estructuralismo de Celso Furtado" en Economía: Teoría y Práctica Nueva Época, No. 10, pp. 55-75.

Mas Colell Andreu, M. Whinston y J. Green (1995) Microeconomic Theory. Nueva York y Oxford: Oxford University Press.

Ocampo José Antonio (2005) Más allá del consenso de Washington: una agenda de desarrollo para América Latina. México, D. F.: ONU.

Petiteville Franck (2002) "Three Mythical Representations of the State in Development Theory" en International Social Science Journal, Vol. 50, No. 155, pp. 115-124.

Prebisch Raúl (1949) Desarrollo económico de América Latina y sus principales problemas, CEPAL, Santiago de Chile. Reimpreso en Girrieri, A. La obra de Prebisch en la CEPAL, Tomo I y II, Lecturas del Trimestre Económico. México, D. F.: Fondo de Cultura Económica, 1982.

Rosenstein-Rodan, Paul N. (1943) "The Problem of Industrialization of Eastern and South-Eastern Europe” Economic Journal, Vol. 53, pp. 202-211.

Sunkel Oswaldo (1972) Capitalismo transnacional y desintegración nacional en América Latina. Santiago: Ediciones Nueva Visión.

Ugarteche Oscar (1986) El estado deudor, economía política de la deuda: Perú y Bolivia 1968-1984. Lima: Instituto de Estudios Peruanos.

Von Neumann John (1937) "Über ein öikonomisches Gleichungssystem und eine Verallgemeinerung des Brouwersschen Fixpunksatzes.” Ergebnisse eines mathematischen Kolloquiums, pp. 73-83. Traducido al castellano como "Un modelo de equilibrio económico general” en Segura J. y Rodríguez C. (Editores) La economía y sus textos. Madrid: Taurus, 1998. 